\title{
LHC and HL-LHC: Present and Future Radiation Environment in the High-Luminosity Collision Points and RHA Implications
}

\author{
Rubén García Alía, Markus Brugger, Francesco Cerutti, Salvatore Danzeca, Alfredo Ferrari, Simone Gilardoni, \\ Yacine Kadi, Maria Kastriotou, Anton Lechner, Corinna Martinella, Oliver Stein, Yves Thurel, Andrea Tsinganis, \\ Slawosz Uznanski
}

\begin{abstract}
-
The High-Luminosity Large Hadron Collider (HL-LHC) is a novel machine configuration which will rely on a number of key innovative technologies to enhance the performance of the present LHC machine as of 2025. The upgrade will also involve increased radiation levels which need to be predicted by combining scaled measurements and calculations in order to define the qualification requirements for electronic systems. In this work we describe such levels first of all by introducing the monitoring and calculation approaches used for the present LHC machine, and secondly by applying scaling factors and dedicated simulations for the future HL-LHC accelerators. We present the levels according to the different areas relevant for the operation of electronics-based equipment, and discuss the associated Radiation Hardness Assurance implications.
\end{abstract}

Index Terms-CERN, Large Hadron Collider (LHC), HLLHC, radiation effects, High Energy Hadron, Monte Carlo methods, FLUKA, RadMON, .

\section{INTRODUCTION}

Single Event Effects (SEEs) and cumulative radiation damage (i.e. Total Ionizing Dose and Displacement Damage) in electronic components and systems can seriously compromise the performance of a high-energy accelerator through unwanted losses of stable beams (known as beam dumps) and/or associated intervention downtime [1]-[3]. In the case of the Large Hadron Collider (LHC, [4]) at the European Organization for Nuclear Research (CERN), such an impact is enhanced by the very large number of units (e.g. power converters, magnet quench protection system, cryogenics) distributed along the $27 \mathrm{~km}$ accelerator and critical to its operation. Such equipment is mostly based on Commercial-OffThe-Shelf (COTS) components due to cost and performance advantages, and therefore needs to be carefully qualified against radiation effects for the relevant field and levels.

The performance of a high-energy accelerator can be evaluated through its capacity of producing beam collisions useful for the high-energy physics community, quantified through the integrated luminosity [5]. The typical unit of integrated luminosity is the inverse femtobarn $\left(\mathrm{fb}^{-1}\right)$ corresponding to roughly $10^{14}$ proton-proton interactions at $\mathrm{TeV}$ energies.

R. García Alía (ruben.garcia.alia@cern.ch), M. Brugger, F. Cerutti, S. Danzeca, A. Ferrari, S. Gilardoni, Y. Kadi, M. Kastriotou, A. Lechner, C. Martinella, O. Stein, Y. Thurel, A. Tsinganis and S. Uznanski are with CERN, CH-1211, Genève, Switzerland.
High Luminosity LHC (HL-LHC) is the future upgrade of the LHC accelerator which aims at delivering an integrated luminosity up to $3000 \mathrm{fb}^{-1}$ over about 10 years of operation, starting from 2025 [6]. The novel machine configuration will rely on a number of key innovative technologies including cutting-edge $11 \mathrm{~T}$ superconducting dipole (bending) magnets and $\mathrm{Nb}_{3} \mathrm{Sn}$ superconducting quadrupole (focusing) magnets, superconducting crab cavities to enhance the beam luminosity, and new collimators and high-energy superconducting links with zero energy dissipation to optimize the position of the power converters.

Even more than for the LHC, the availability of the HL-LHC accelerator will be critical to its successful performance. This is partially related to the so-called leveling of the luminosity in the accelerator [6]. In the LHC (operated without leveling at the high luminosity interaction points of ATLAS and CMS) the decrease in luminosity as a function of time is related mainly to the loss of particles in the interaction points from collisions. This is also the reason why fills are dumped after a certain time period due to the quality loss of the beam.

However, in the case of HL-LHC, the large peak luminosity would lead to a too high collision rate for the experiment detectors to process, in an effect known as pile-up. In order to mitigate this effect while achieving the desired integrated luminosity, the luminosity is leveled to a value below its peak performance until it starts to suffer from the effect of burn-off (e.g. after roughly 8 hours according to optimization calculations) and after which the beam is eventually dumped. Due to the fact that the leveled integrated luminosity is only larger than the non-leveled one after a certain fill duration, HL-LHC will require relatively long fills to operate in an efficient manner, thus tolerating a very low number of radiation induced dumps for an efficient operation. This, in combination with the higher radiation levels expected that will be described in this work, the state-of-the-art microelectronic technologies to be introduced and the more complex equipment sets a very challenging scenario in terms of radiation qualification. Therefore, the purpose of this work is to evaluate the expected radiation environment in the critical points of the HL-LHC and the associated impact on the Radiation Hardness Assurance (RHA) of the critical systems. 


\section{RADiATION LEVEL MONITORING}

\section{A. LHC layout}

Before presenting the results of the radiation levels in the LHC and its vicinity, it is important to clearly understand the basic layout of the machine [4]. The LHC is composed of eight arcs and eight straight sections also known as Insertion Regions (IRs). The latter are each $528 \mathrm{~m}$ long, with four of them hosting collision points and detectors (IR1, IR2, IR5 and IR8), and the rest used for machine utilities: radio frequency cavities (IR4), collimation (IR3 and IR7) and beam dumps (IR6). They layout of the full LHC ring is depicted in Fig. 1.

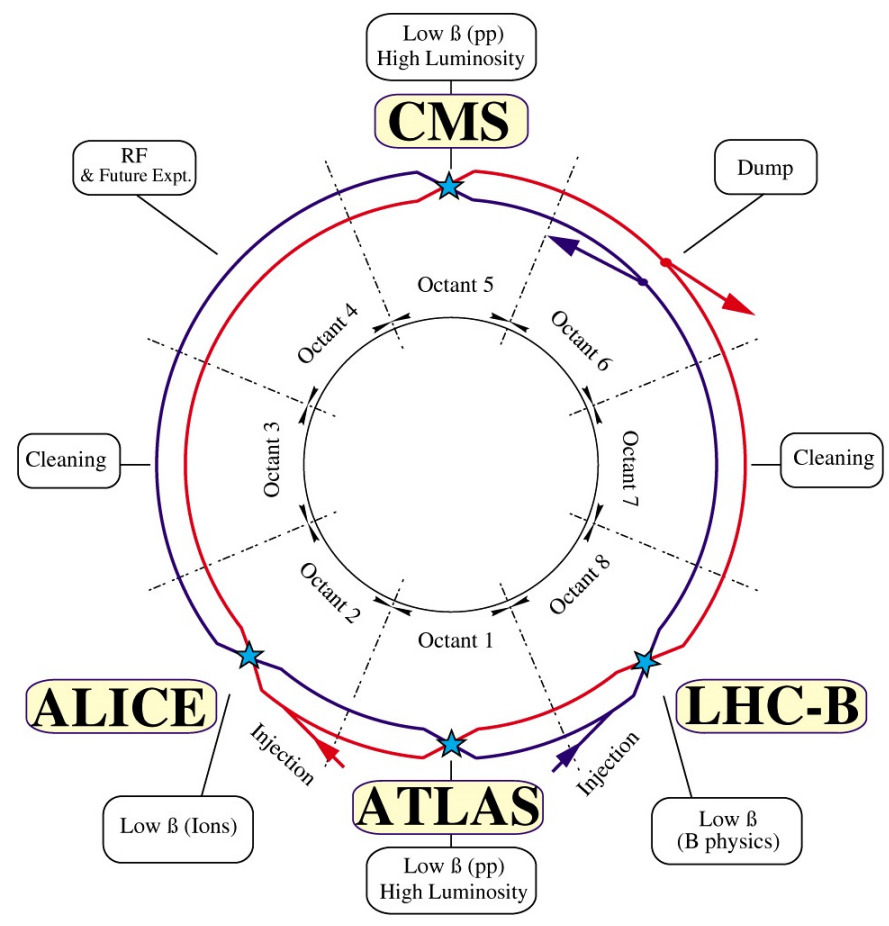

Fig. 1. Schematic representation of the LHC layout showing the eight octants and IRs. Beam 1 circulates clockwise, and Beam 2 anticlockwise.

Each of the eight LHC arcs between two IRs contains 42 half-cells. A half-cell is the periodic part of the LHC arc lattice, each consisting of a string of three twin aperture main dipole magnets (MB.A, MB.B and MB.C) and one short straight section hosting one quadrupole (MQ). The half-cell length is $54 \mathrm{~m}$ long and is shown schematically in Fig. 3 . The arc occupies half-cells from 14 to 34, both included and on each side of the IR. Each LHC arc has one Dispersion Suppressor (DS) on each end, acting as a transition between the LHC arcs and Insertion Regions (IR) and consisting of four individually powered quadrupole magnets each separated by two dipole magnets. The DS aims at reducing the machine dispersion close to the IRs. The DS occupies half-cells from 8 to 13 , both included. In addition, the quasi-straight sections between the two DS of an IR are called Long Straight Sections (LSS, half-cells 1-7). The LSS can be further subdivided into the matching section (arrangement of quadrupole magnets, half-cells 4-7) and the inner triplet (IT, half-cells 1-3). The IT is an assembly of three quadrupole magnets used to reduce the optical $\beta$-functions at the Interaction Points (IPs). The LHC has triplet assemblies in IR1, IR2, IR5 and IR8, corresponding to the four collision points. A representation of the different areas and corresponding half-cell numbering is shown in Fig. 2

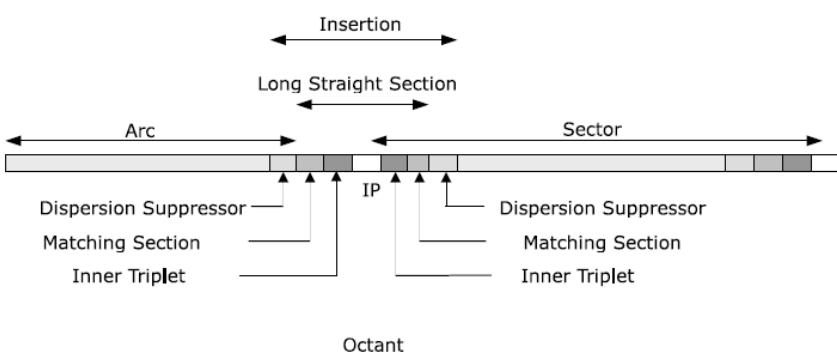

Fig. 2. LHC regular octant layout, with the inner triplet occupying half-cells from 1-3, the matching section from 4-7, the DS from 8-13, and the arc from $14-34$

This work will focus on the radiation levels in the vicinity of Interaction Points (IP) 1 and 5, which are critical owing to their very large luminosity and associated collision debris. However, other points of the accelerator not covered in this work (e.g. the collimation region in IP7 [7]) are also highly critical in terms of radiation levels and potential impact to electronics.

Finally, there are service alcoves constructed adjacent the LHC tunnel and designed to host large quantities of accelerator equipment nearby tunnel regions with radiation levels too large to host active COTS components. Their topology will be further discussed in the context of the location of the radiation monitors.

\section{B. Radiation source term in high-luminosity interaction points}

The mixed-radiation field in the vicinity of the LHC highluminosity interaction points (IP1 and IP5) is dominated by the particle debris of proton-proton inelastic interactions, which generate secondary particles with average multiplicities of 120 per single proton-proton collision. The prevalent secondary particles at $5 \mathrm{~mm}$ from the interaction point are photons $(\sim 50 \%)$ and charged pions $(\sim 35 \%)$. Whereas most of these particles are intercepted by the detectors, a small fraction of them are emitted at very small angles with respect to the beam, thus reaching accelerator elements.

In addition to the direct luminosity debris, halo particles caught in the so-called debris collimators around the experiments and initiating hadronic and electromagnetic showers are also an important source of radiation for the accelerator equipment. This is also the case in the dedicated collimation points of the LHC (IR3 and IR7) which especially in the case of the latter and though not treated explicitly in this work, also exhibit very large radiation levels. In any case, for the areas of interest to the operation of electronic equipment, IR1 and IR5 represent the most stringent radiation environment conditions for the HL-LHC machine, and are therefore the subject of this study.

In order to minimize the beam size and thus maximize the luminosity, the HL-LHC upgrades foresee changes in the IR1 


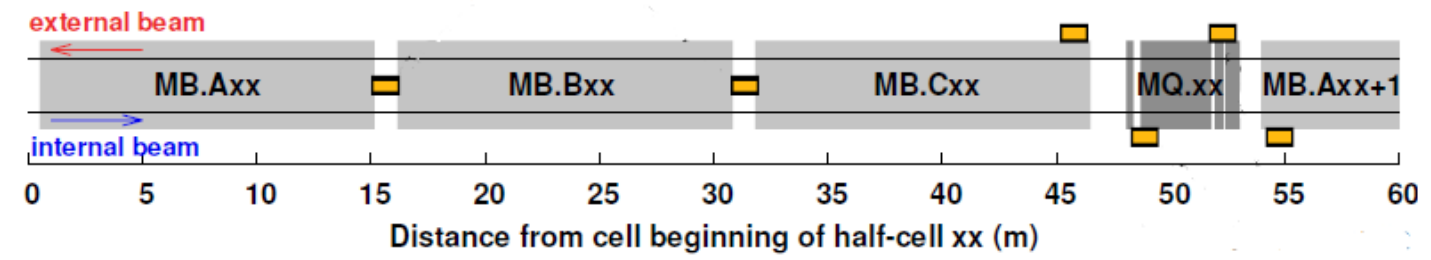

Fig. 3. Schematic representation of an LHC arc half-cell including the standard BLM monitor positions in yellow.

and IR5 matching sections, involving larger physical apertures. As a consequence of this, the number of debris particles entering the matching section per primary collision is much larger than in the case of the current machine. In order to protect the cold magnets in the matching section from the collision debris, a network of so-called Target Long Physics Debris collimators (TCLs) is present. These are named TCL4, 5 and 6 depending on the matching section half-cell in which they are installed. For the current machine, not all TCLs are necessary to protect the magnets, however for HL-LHC all of the TCLs are indispensable due to the larger amount of debris particles. As will be later shown, this has significant implications on the associated radiation levels.

\section{Radiation Monitoring and Levels}

1) LHC tunnel: The LHC beam loss monitoring (BLM) system [8], [9] is based on ionizing chambers and has the main purpose of preventing the superconducting magnets from quenching as well as of protecting the machine components from damage. About 4000 of them are installed in the LHC. Despite their main use as prompt beam loss detectors triggering a beam dump when thresholds are passed, the BLM signals can also be integrated in time and used to monitor the TID levels around the accelerator tunnel. In a standard LHC arc half-cell, BLMs are configured as shown in Fig. 3.

Measurements of the integrated BLM TID levels during the 2016 proton-proton runs (corresponding to an integrated luminosity of $40 \mathrm{fb}^{-1}$ ) are shown in Fig. 4 for IP1 and IP5, and are reported in other LHC locations in [10]. As can be seen, different regions can be identified according to their radiation levels. In the IR, annual TID values at the BLM locations can be as large as $10 \mathrm{kGy}$ and therefore the use of commercial electronics is excluded. The same applies to the DS in which peaks of roughly $100 \mathrm{~Gy} / \mathrm{yr}$ are reached, however the use of qualified radiation tolerant systems based on COTS components can be exceptionally considered for locations below the dipole magnets, reaching BLM levels of roughly $10 \mathrm{~Gy} / \mathrm{yr}$. In the LHC arc region, radiation levels are in general dominated by beam-gas interaction and with the exception of several peaks in the odd half-cells near the DS are below $1 \mathrm{~Gy} / \mathrm{yr}$ and therefore acceptable for the use of COTS from a cumulative radiation damage lifetime perspective. However, the situation is very different for SEE failure risk, which increases according to the number of deployed units in the arc and therefore needs to be carefully considered.

The RadMON is a dedicated monitoring system fully developed, maintained and operated in the scope of the Radiation to
Electronics (R2E) project at CERN [11]-[13]. Currently there are over 300 units installed in the LHC accelerator. Through the calibration of its COTS-based detectors, the three quantities relevant to radiation damage can be measured by a PIN diode (Displacement Damage), RadFET (Total Ionizing Dose) and SRAM SEU monitor (Equivalent High-Energy Hadron fluence, represented as $\mathrm{HEH}_{\mathrm{eq}}$ and defined as the fluence of hadrons above $20 \mathrm{MeV}$ plus a weighted contribution from neutrons in the $0.2-20 \mathrm{MeV}$ range according to a generalized SEU response [14]). RadMON detectors in the LHC tunnel are typically placed below the magnets shown in Fig. 3, roughly $70 \mathrm{~cm}$ below beam height and in regions with approximately a factor 3 lower radiation levels than BLMs at a similar longitudinal position.

The measured proton-proton 2016 (for $40 \mathrm{fb}^{-1}$ ) $\mathrm{HEH}_{\mathrm{eq}}$ fluence values are shown in Fig. 5 for the right side of IP5 as a function of the distance to it, also including the BLM data. Note that two different y-axes are used, with a relation of $5 \times 10^{8} \mathrm{HEH}_{\mathrm{eq}} / \mathrm{cm}^{2}$ per Gy. As can be seen, both data sets are consistent in terms of longitudinal distribution. As a comparison with other well known environments, the annual $\mathrm{HEH}_{\mathrm{eq}}$ fluence is roughly $1-2 \times 10^{5} \mathrm{~cm}^{-2}$ at ground level and $3 \times 10^{9} \mathrm{~cm}^{-2}$ for a $98^{\circ}, 800 \mathrm{~km}$ Low-Earth Orbit (LEO).

As can be seen, the radiation levels are clearly larger in the odd half-cells 9, 11 and 13. This can be attributed to the large optical dispersion functions present in these half-cells which induce losses from off-momentum protons. As can be seen in Fig. 4, this is generally the case on both sides of IP1 and IP5 and also partially affects half-cell 15 .

As mentioned above, the debris collimator settings near the high-luminosity experiments play a crucial role in the radiation levels both in their vicinity (e.g. the RR alcoves, as will be detailed below) and in regions further downstream, such as the DS. As further detailed in [15], this effect is represented in Fig. 6 which shows the BLM dose values to the right of IP5 normalized to the luminosity for two periods of operation, one with the TCL5 closed and TCL6 open (red curve), and one with the opposite configuration (blue curve), typically applied when the forward physics experiments are operated near that location. As can be seen, closing TCL6 increases the radiation levels in the $\mathrm{RR}$ alcove, hosting a large quantity of electronic systems, but also contributes to reducing the radiation load in the DS. Therefore, this is an example of how the accelerator settings can impact the radiation levels, thus requiring a careful optimization analysis, involving not only radiation to electronics considerations but also other aspects such as primarily the machine protection (e.g. energy 


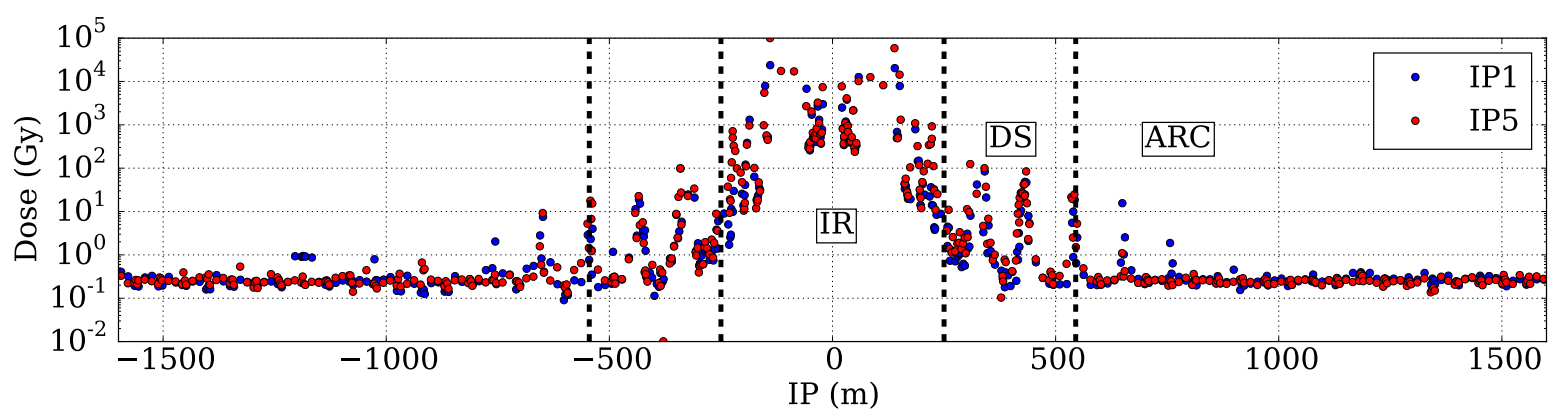

Fig. 4. BLM TID (dose in $\mathrm{N}_{2}$ ) values in IP1 and IP5 during the 2016 proton-proton run.

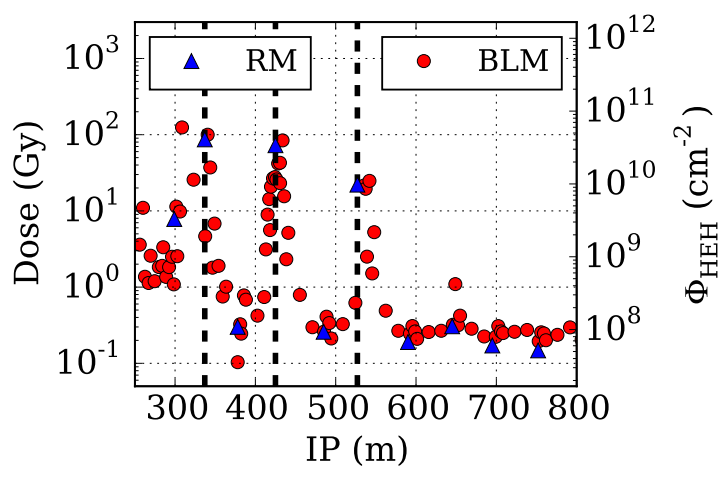

Fig. 5. $\mathrm{HEH}_{\mathrm{eq}}$ fluence for the 2016 proton-proton run to the right of IP5 as measured by the RadMON (RM) system together with the BLM TID (dose in $\mathrm{N}_{2}$ ) measurements. The vertical lines represent, from left to right, half-cells 9, 11 and 13 .

deposition in the magnets).

2) Shielded Areas: As mentioned above, the LHC tunnel areas in the IR and parts of the DS have radiation levels that are in general too large to be able to host systems based on COTS components. For this reason, shielded service galleries were constructed in the vicinity of the tunnel to locate the necessary hardware. The UJ (junction chamber) and UL (liaison gallery between underground works) zones are heavily shielded areas near the collision points, whereas the RRs are lightly shielded near the IR/DS limit.

A schematic representation of the RadMON location in the UJ, UL and RR alcoves and their vicinity is shown in Figs. 7 and 8 for the area right of IP1. The left side is symmetric to it and in IP5 the UJ/UL configuration is different to IP1 but will not be treated here in detail owing to the fact that the sensitive electronic equipment was relocated from these locations during the Long Shutdown 1 (LS1) as a part of the R2E mitigation strategy. As can be seen, several tens of $\mathrm{cm}$ of concrete and/or cast iron separate the tunnel from the shielded areas.

The annual HEH fluences for the proton-proton run in 2016 in the shielded areas shown in Figs. 7 and 8 are presented in Table II. Especially relevant in terms of operational impact are the radiation levels in the RRs, which as will be detailed below host a large quantity of power converters that can suffer
TABLE I

ANNUAL HEH FLUENCE IN UNITS OF $\mathrm{cm}^{-2}$ AS MEASURED BY THE RADMON SYSTEM DURING THE 2016 PROTON-PROTON OPERATION, CORRESPONDING TO $40 \mathrm{fb}^{-1}$. WITH THE EXCEPTION OF UJ56, THE SHIELDED LOCATIONS HOST A LARGE QUANTITY OF LHC QUALIFIED EQUIPMENT BASED ON COTS COMPONENTS.

\begin{tabular}{c|c|c} 
Location & Tunnel & Shielded \\
\hline \hline RR13 & $1.36 \times 10^{9}$ & $4.19 \times 10^{7}$ \\
RR17 & $1.00 \times 10^{9}$ & $5.37 \times 10^{7}$ \\
RR53 & $2.87 \times 10^{9}$ & $1.17 \times 10^{8}$ \\
RR57 & $4.55 \times 10^{9}$ & $1.56 \times 10^{8}$ \\
\hline UL14 & - & $1.27 \times 10^{7}$ \\
UL16 & - & $1.66 \times 10^{7}$ \\
\hline UJ14 & $2.64 \times 10^{11}$ & - \\
UJ16 & $2.79 \times 10^{11}$ & - \\
UJ13 & - & $8.30 \times 10^{8}$ \\
UJ17 & - & $4.12 \times 10^{8}$ \\
UJ56 & - & $6.65 \times 10^{8}$ \\
\hline
\end{tabular}

TABLE II

EXPECTED ANNUAL RR FLUENCES FOR $50 \mathrm{fb}^{-1}$ AS A FUNCTION OF THE TCL6 SETTINGS AND DERIVED FROM 2016 MEASUREMENTS IN RR53 AND RR57. IN THE CASES OF THE SHIELDED AREAS AND TCL6 OPEN, THE NUMBER OF SEU COUNTS PER RADMON AND SETTING CAN BE AS LOW AS $\sim 10$, THEREFORE A LARGE STATISTICAL UNCERTAINTY APPLIES.

\begin{tabular}{c|c|c} 
TCL6 setting & Tunnel & Shielded \\
\hline \hline Open & $1.03 \times 10^{9}$ & $3.85 \times 10^{7}$ \\
Closed & $1.08 \times 10^{10}$ & $3.65 \times 10^{8}$ \\
\hline
\end{tabular}

from SEE failures. As can be seen in Fig. 9 the radiation levels measured by the RadMON outside the RR (i.e. on the tunnel side) have a very strong correlation with the TCL settings, as shown in Fig. 6 for the BLMs. As can be seen in Fig. 9 for the RadMON measurements, the radiation levels in the RR change by roughly a factor 10 depending on the TCL settings. The values extracted from the 2016 measurements in the RRs of IP5 for the two different TCL collimator settings are shown in Table II.

\section{RADIATION LEVEL SimULATIONS}

The radiation levels in the $\mathrm{LHC}$ are simulated using the FLUKA Monte Carlo code [16], [17]. This requires a highly detailed description of the complex accelerator geometry as well as the associated beam optics. FLUKA calculations are used in a very broad variety of high-energy accelerator applications, ranging from energy deposition studies associated to superconducting magnet quenches and material degradation, 


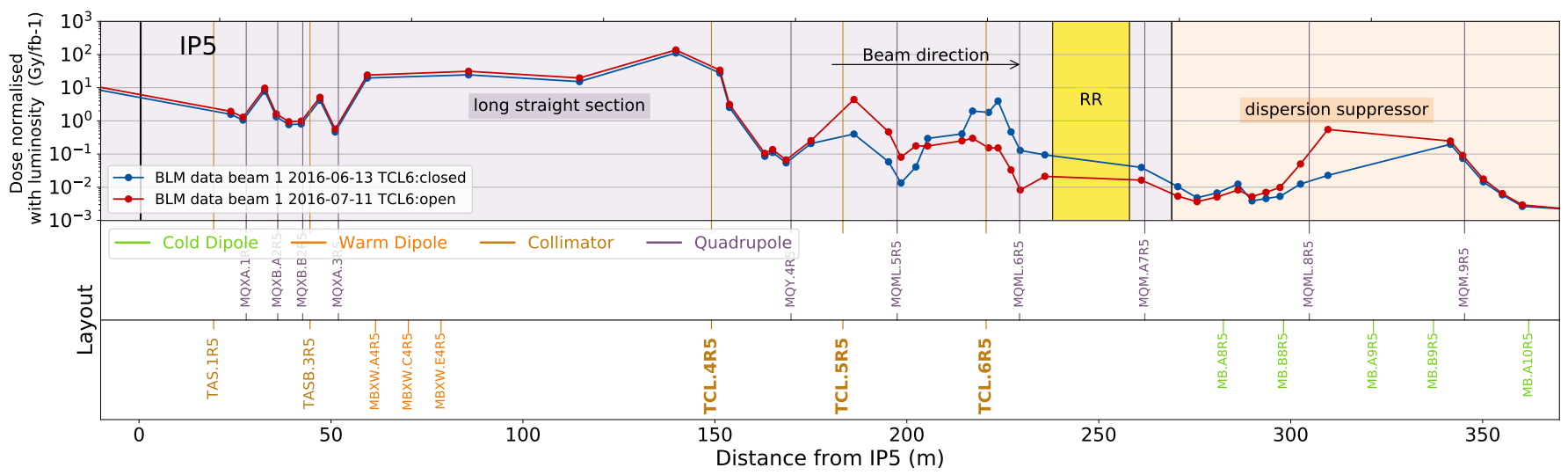

Fig. 6. BLM TID (dose in $\mathrm{N}_{2}$ ) readings to the right of IP5 during two different 2016 proton-proton periods, normalized to the respective integrated luminosity, and for two different TCL settings.

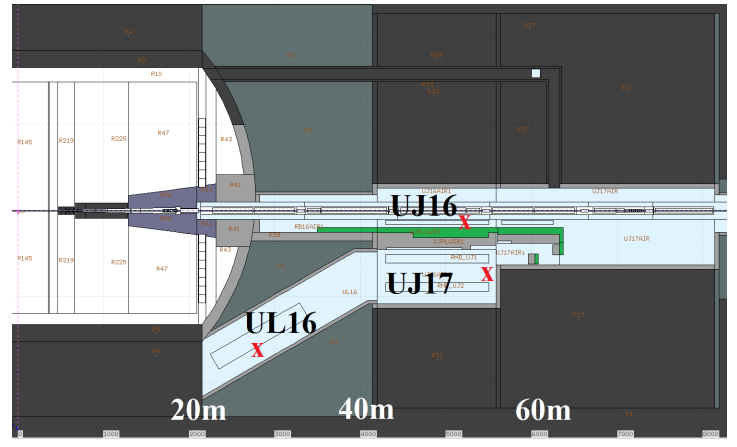

Fig. 7. FLUKA top-view geometry of the HL-LHC layout right of IP1, indicating the UL and UJ locations as well as the respective RadMONs, marked with red crosses. The distance to the IP is also included.

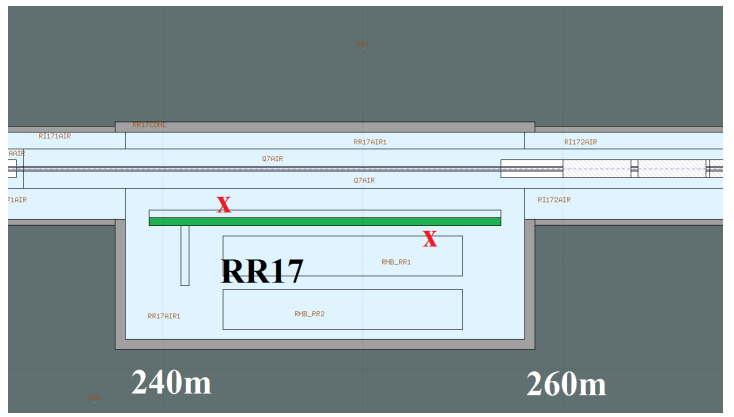

Fig. 8. FLUKA top-view geometry of the HL-LHC layout right of IP1, indicating the RR location as well as the respective RadMONs, marked with red crosses. The distance to the IP is also included. The situation in RR13, RR53 and RR57 is analogous.

through layout and shielding optimization, and to radiation to electronics studies. Previous simulations and measurement benchmarks in the radiation damage to electronics context for the present LHC machine can be found in [18], [19].

In the case of the regions in the vicinity of the interactions points (e.g. IP1, IP5) the radiation environment is dominated by particle collision debris. The latter can come in the form of particles directly produced in the collisions or of offmomentum protons that are intercepted by the collimation system or in the Dispersion Suppressor (DS) generating EM and hadronic showers which originate the mixed-radiation

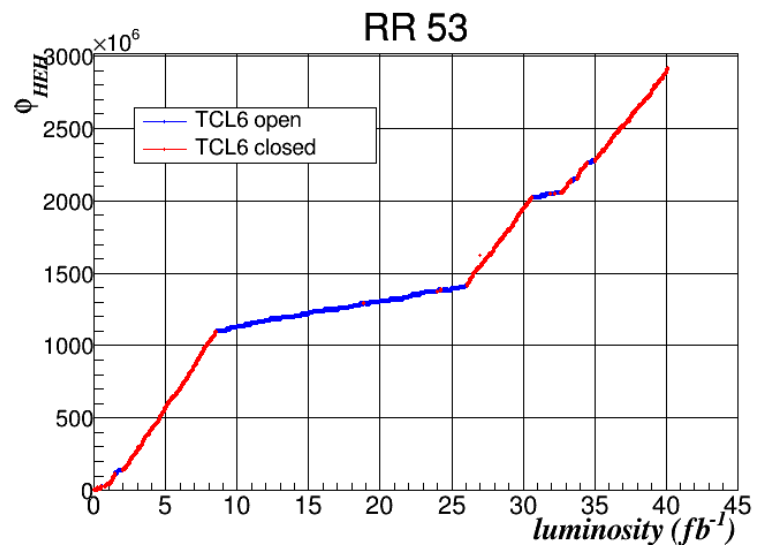

Fig. 9. $\mathrm{HEH}_{\mathrm{eq}}$ fluence as a function of luminosity as measured by the RadMON outside the RR (see Fig. 8) for different TCL settings.

field responsible for the failure and degradation of electronic equipment.

As was done for the present LHC case, the HL-LHC radiation levels are analyzed separately for the tunnel and shielded areas.

\section{A. HL-LHC tunnel}

The calculated TID (in air) and $1 \mathrm{MeV} \mathrm{n}_{\mathrm{eq}}$ radiation levels in the HL-LHC LSS tunnel section for IR1 and IR5 are shown in Fig. 10 as a 1D cut at beam height and $1.6 \mathrm{~m}$ away from the beamline, and corresponding to the full HLLHC lifetime. Whereas the levels, reaching values above $100 \mathrm{kGy}$ and $10^{15} \mathrm{n}_{\mathrm{eq}} / \mathrm{cm}^{2}$, clearly exclude the use of COTS components as a baseline approach, some applications such as beam monitoring or magnet protection systems require active electronics operating in such locations. This can be the case for instance of the superconducting magnet protection diodes operating at cryogenic temperatures and used as bypass elements in case of a magnet quench [20]. The latter degrade due to displacement damage leading to an increased forward bias voltage and potential permanent thermal damage. Therefore, a detailed study of the expected radiation levels and associated qualification is clearly required. 
Moreover, a benchmark of the BLM radiation levels in the LSS of IP1 is shown in Fig. 11 for the TCL6 closed condition, exhibiting an excellent agreement between simulated and measured values.

A transversal cut of the $1 \mathrm{MeV} \mathrm{n}_{\mathrm{eq}}$ fluence is shown in Fig. 12 for the location immediately downstream the D1 dipole magnet, which as shown in Fig. 10 corresponds to a local minimum in the longitudinal dose distribution. As can be seen, the levels at the tunnel walls are still $10^{14} \mathrm{n}_{\mathrm{eq}} / \mathrm{cm}^{2}$ and only roughly a factor $\sim 3$ reduction is obtained with respect to the area immediately outside the magnet cryostat. In terms of dose, values range from $\sim 15 \mathrm{kGy}$ near the magnets to $\sim 5 \mathrm{kGy}$ at the tunnel walls.

As to what concerns the tunnel radiation levels in the DS, explicit calculations for HL-LHC will be carried out in the near future. Such simulations rely on a two-step approach in order to obtain statistically meaningful energy deposition and radiation level results in the DS. In the first step, including the full geometry from the radiation source term (i.e. the pp collisions), the protons lost in the DS beam pipe are scored, and then propagated in the second step simulation focusing on the DS area.

However, in first approximation levels can be scaled with luminosity from values for the present machine. The assumed scaling is based on the fact that losses in this area are due to off-momentum protons from the proton-proton primary collisions in the experiments. Fig. 13 shows the simulated versus measured dose levels in a fill during 2016, normalized per collision. An inverse femtobarn $\left(\mathrm{fb}^{-1}\right)$ corresponds to $8 \times 10^{13}$ collisions, therefore the values can be scaled up by a factor $2.4 \times 10^{17}$ in order to consider the expected integrated luminosity of $3000 \mathrm{fb}^{-1}$ for the full HL-LHC lifetime.

Therefore, peaks would correspond to HL-LHC lifetime doses of roughly $100 \mathrm{kGy}$ at the BLM locations, and are reached in the vicinity of the quadrupole magnets. For locations near the dipole magnets (MBs) hosting the vast majority of the electronics racks in the DS, the maximum BLM level is reached towards the end of the BB11 magnet, and corresponds to roughly $10^{-14} \mathrm{~Gy} /$ collision, or $2.4 \mathrm{kGy}$ for the HL-LHC lifetime. In addition, for the dipole magnets in half-cells 8 and 9 , the values are roughly a factor 10 lower. The electronic equipment in this area is placed below the magnets, and therefore somewhat less exposed to the radiation field. As mentioned above, a factor 3 reduction in the radiation level from the BLM to the RadMON/equipment area is considered. Therefore, the TID values below the dipoles for the HL-LHC lifetime would correspond to $\sim 80$ Gy in half-cells 8 and 9 , and $\sim 800$ Gy towards the end of half-cell 11 . Thus, most of the areas below the dipoles in the HL-LHC DS are expected to have annual TID levels below $10 \mathrm{~Gy} /$ year.

\section{B. HL-LHC shielded areas}

The results of the calculated $\mathrm{HEH}_{\mathrm{eq}}$ fluences for the region right of IP1 and up to $260 \mathrm{~m}$ from the IP are shown in Fig. 14 as a 2D top-view map. Results are normalized to one year of nominal HL-LHC operation $\left(250 \mathrm{fb}^{-1}\right)$ and correspond to the averaged value at $\pm 10 \mathrm{~cm}$ from the beam height.
As can be seen, the radiation levels in the $\mathrm{RR}$ alcove are dominated by the TCL collimation system directly upstream of it. As opposed to the LHC case, for HL-LHC the presence of all of the TCLs is indispensable for magnet protection [6]. In addition to the impact of the TCLs, other factors such as the larger crossing angle or larger upstream aperture also contribute to an increased particle debris and radiation levels in the HL-LHC scenario. As can be seen, the annual levels in the UJ and RR shielded areas can be as large as $5 \times 10^{9} \mathrm{~cm}^{-2} \mathrm{HEH} / \mathrm{cm}^{2}$.

Therefore, it is important to note that whereas in the regions in the direct vicinity of the interaction points (e.g. UJ, UL) the HL-LHC radiation levels are expected to increase proportionally to the integrated luminosity with respect to the present LHC machine, the levels in other areas such as the $\mathrm{RR}$ are expected to have a more complicated behavior and dependence with the accelerator settings.

\section{RHA IMPLICATIONS}

The vast majority of standards for radiation hardness assurance (i.e. such as those included in [21] for TID effects) are developed for space applications and are therefore not directly applicable in the high-energy accelerator context due to (i) the different radiation environment (ii) the different criticality levels, with failures in the accelerator environment being tolerable if they do not significantly impact the performance of the machine and (iii) the system-level as opposed to component level focus of radiation hardness assurance of highenergy accelerator equipment due to the very large number of active semiconductor components and amount of system units involved. However, space standards serve as an essential input for the qualification approach of LHC equipment.

In the LHC machine, systems for the accelerator powering, controls, protection and monitoring are generally based on Commercial-Off-The-Shelf (COTS) components due to performance, availability and cost constraints. Therefore, such parts can suffer from radiation effects, mainly through SEE failures and TID degradation. A typical LHC system design such as that in charge of controlling the power converters providing current to the magnets [22] will contain a broad variety of components potentially sensitive to radiation, including Field-Programmable Gate Arrays (FPGAs), Analog-to-Digital Converters (ADCs), operational amplifiers, voltage regulators, memories, opto-couplers and others.

Therefore, the Radiation Hardness Assurance (RHA) at CERN [22] is first of all based on the selection of components the fulfill the electrical requirements of the system design, and for which if possible historical radiation qualification data is available either in the CERN/R2E parts database, or the literature. In the absence of representative radiation data, type testing is carried out on the part according to the radiation levels expected for its operational conditions. If the type testing does not fulfill the radiation tolerance requirements, an alternative part previously identified is tested. If no suitable substitute is found, the system design might need to be altered in order to be based on compliant components.

Once the type testing is successfully completed, the component is procured in large quantities (typically batches of 

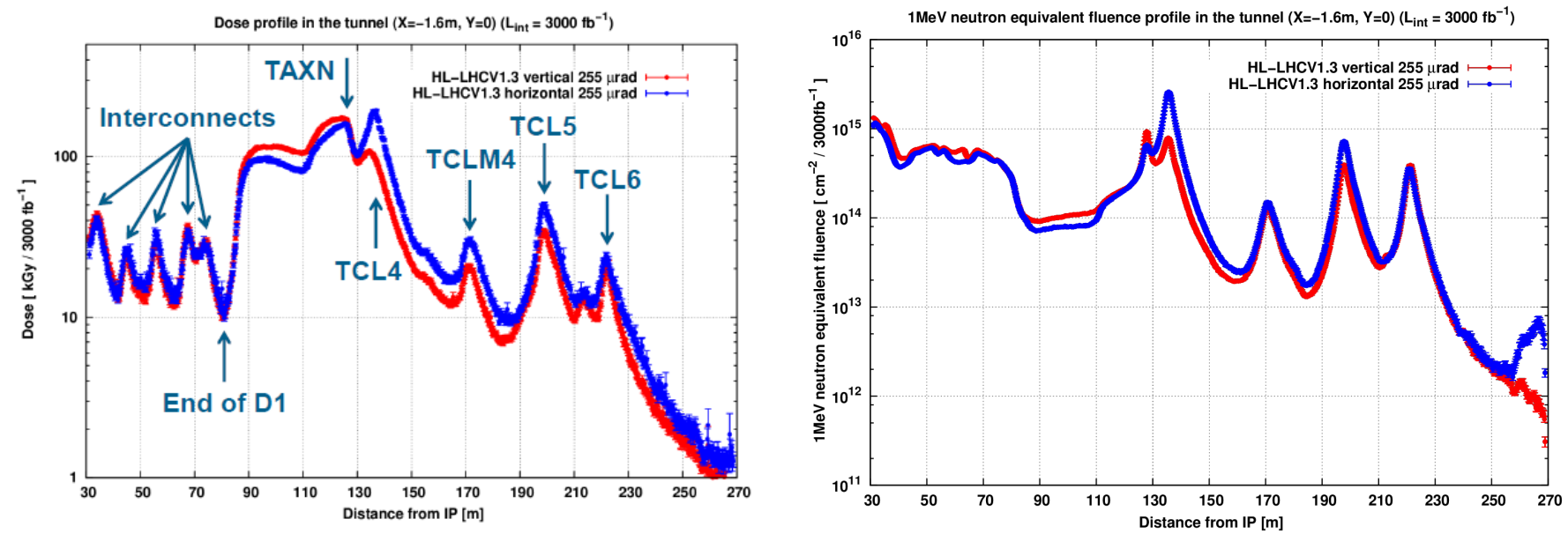

Fig. 10. Calculated dose in air and $1 \mathrm{MeV}_{\mathrm{eq}}$ profile in the HL-LHC tunnel at beam height and $1.6 \mathrm{~m}$ from the beam, for the full $3000 \mathrm{fb}^{1}$ expected lifetime Simulations include both horizontal (IP5) and vertical (IP1) beam crossing, a crossing angle of $255 \mu$ rad and optics version 1.3. The TAXN corresponds to an absorber for neutral collision debris.

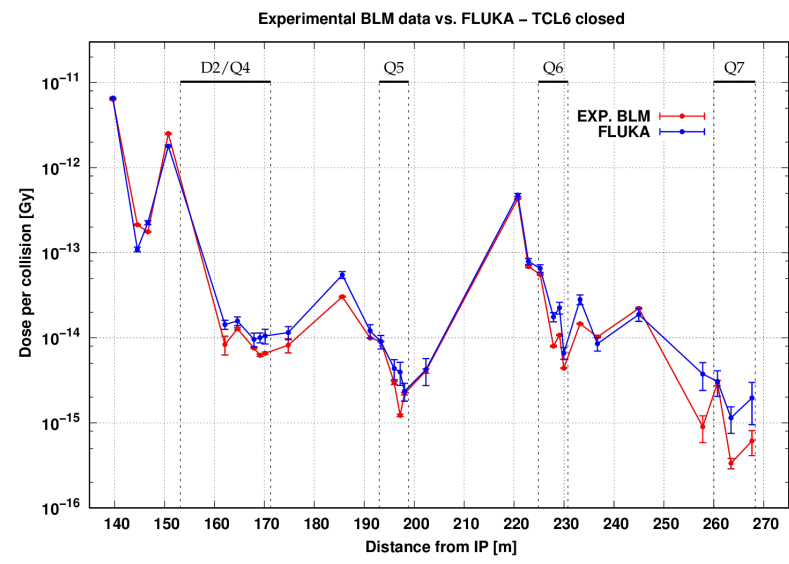

Fig. 11. Simulated and measured normalized BLM radiation levels (dose in $\mathrm{N}_{2}$ ) for the present LHC LSS, with TCL6 closed.

thousands of units). Each batch is individually qualified to confirm the conformity with the previously performed type tests and to assure the conformity of the component radiation response within the batch. When possible, all production components for a given system should be procured from a single fabrication lot to decrease the component-to-component variability to the intra-lot (as opposed to inter-lot) spread. When possible, a single lot can be used transversally for different systems. The batch testing strategy typically requires a minimum of 10 irradiated components and one reference per batch.

The type and batch testing are typically carried out in cobalt-60 sources for TID and high-energy proton facilities for SEE and TID. Though typically not a dominating effect, displacement damage can also be evaluated using protons and/or $14 \mathrm{MeV}$ neutrons. In addition, the CHARM facility at CERN [23] can also be used for such purpose, provided the representativeness of its radiation field, as well as its very large irradiation volume, enabling the characterization of a broad set

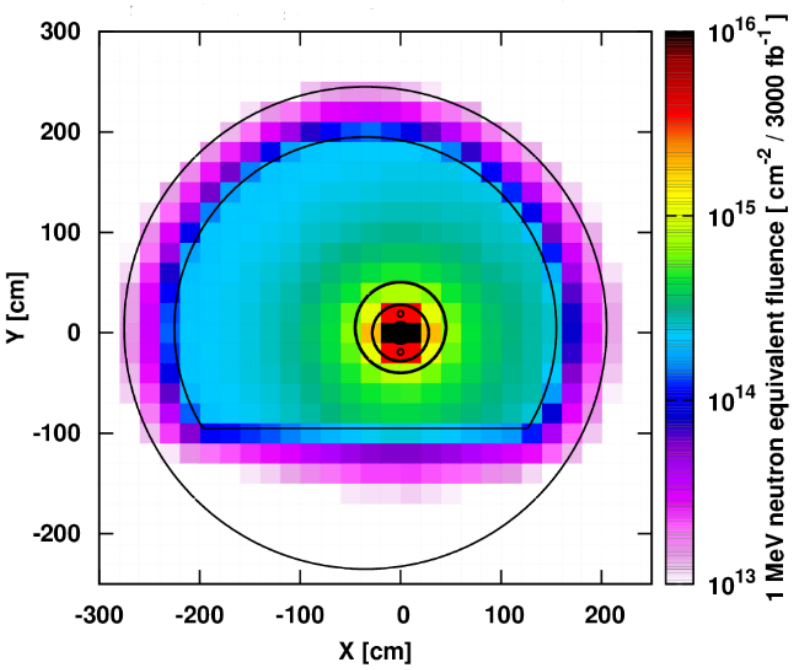

Fig. 12. 2D transversal cut of the $1 \mathrm{MeV} \mathrm{n}_{\mathrm{eq}}$ fluence at the $\mathrm{D} 1$ longitudinal location (see Fig. 10).

of components in parallel.

After the batch testing, and once a pre-series of the system is produced, the latter is qualified at a system level in the CHARM facility, again for the TID, DD and SEE cross section limits imposed by its respective lifetime and maximum tolerated failure rate, respectively. As described above, measurements and simulations are used in combination in order to predict the radiation levels for the HL-LHC era in the various locations of interest. The knowledge of the radiation levels is also used to implement mitigation strategies for lifetime constraints related to cumulative radiation damage through the preventive substitution or rotation of the equipment during planned interventions.

Table III summarizes the expected annual HL-LHC radiation levels as a function of the various accelerator locations relevant for the operation of systems based on COTS compo- 


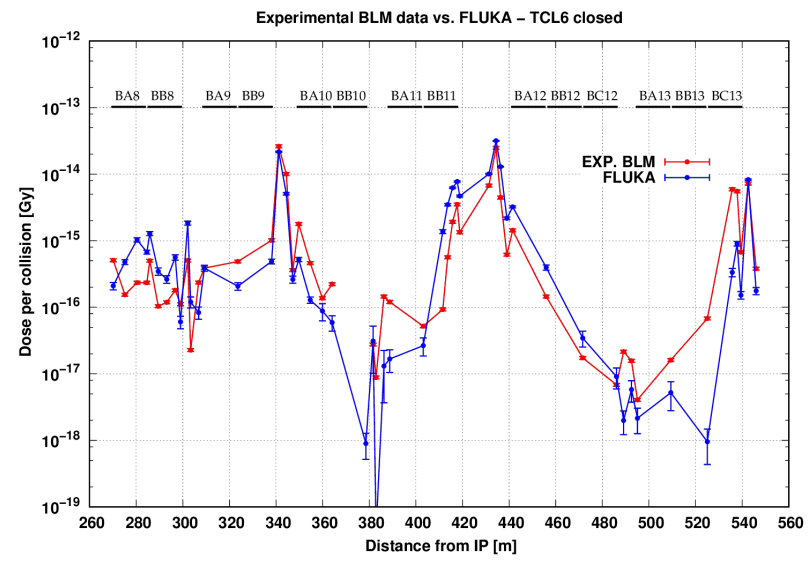

Fig. 13. Simulated and measured normalized BLM radiation levels (dose in $\mathrm{N}_{2}$ ) for the present LHC DS.

nents. For the DS, the peak value below the dipole magnets towards the end of half-cell 11 is considered.

In addition, it is to be noted that for the ARC, levels are scaled with integrated beam intensity from values measured in the present machine, however they will strongly depend on the vacuum level in the HL-LHC machine, which is a parameter very difficult to estimate and control. Furthermore, such predicted values correspond to the expected baseline from beam-gas interactions, however localized radiation level peaks due to other sources cannot be excluded and will need to be carefully monitored.

In addition to the evaluation of the radiation levels, it is necessary to define the number of acceptable radiation failures in order not to compromise the performance of the HL-LHC machine. As shown in Fig. 15 and further detailed in [6], the considered limit for HL-LHC is 0.1 radiation induced dumps per $\mathrm{fb}^{-1}$, or 25 dumps per nominal operation year. Considering the large amount of system types potentially sensitive to radiation and operating in locations with significant $\mathrm{HEH}_{\mathrm{eq}}$ levels, it is reasonable to consider a limit of one failure per system type and year, though detailed individual studies are required in order to evaluate this more precisely. In addition to the possible SEE impact, lifetime limitations due to TID and DD also need to be carefully considered.

As an example of the implications of such levels on the RHA strategy, one of the critical power converter system for HL-LHC (the 4-6-8 kA power converters for $8 \mathrm{~V}$ ) will have 30 units in RR13/17 and another 30 units in RR53/57, thus requiring to be qualified for a system SEE cross section of less than $5 \cdot 10^{-12} \mathrm{~cm}^{2} /$ unit in order to comply with a one failure per nominal operation year limit; as well as TID and DD limits of $\sim 60$ Gy and $\sim 3 \times 10^{11} \mathrm{n}_{\mathrm{eq}} / \mathrm{cm}^{2}$ to guarantee a lifetime compatible with that of the machine (e.g. 12 years for the case of HL-LHC). Such tests are carried out under an experimental configuration that will reproduce the spectral hardness the system will encounter in operation, in order to account for possible SEE cross section dependencies with energy [24].

Moreover, systems installed under the main superconducting dipole magnets (MBs) in the DS of the present machine have in general been qualified up to TID limits of $200 \mathrm{~Gy}$.
TABLE III

EXPECTED ANNUAL $\left(250 \mathrm{fb}^{-1}\right)$ RADIATION LEVELS FOR HL-LHC IP1 AND IP5. FOR THE DS, ONLY AREAS BELOW THE DIPOLE MAGNETS ARE CONSIDERED.

\begin{tabular}{c|c|c|c}
\multirow{2}{*}{ Location } & HEH fluence & $1 \mathrm{MeV} \mathrm{n}_{\text {eq }}$ & TID \\
\cline { 2 - 4 } & \multicolumn{2}{|c}{$\left(\mathrm{cm}^{-2}\right)$} & \multicolumn{1}{c}{ (Gy) } \\
\hline \hline UJ & $5 \times 10^{9}$ & $5 \times 10^{10}$ & 10 \\
UL & $10^{8}$ & $10^{9}$ & 0.2 \\
RR & $3 \times 10^{9}$ & $3 \times 10^{10}$ & 6 \\
\hline DS & $5 \times 10^{10}$ & $5 \times 10^{10}$ & 100 \\
ARC & $10^{9}$ & $10^{9}$ & 2 \\
\hline
\end{tabular}

Therefore, the expected maximum levels of roughly $1 \mathrm{kGy}$ during the full HL-LHC operation will imply the need of either mitigating (through relocation and/or substitution) and/or preventing (through the design of radiation-tolerant COTS-based systems) the possible negative impact from radiation.

In CHARM, levels of up to $200 \mathrm{~Gy}$ can be reached in one week of operation, therefore covering the lifetime of most HL-LHC applications. As to what regards the HEH-equivalent fluence, weekly values of $\sim 5 \times 10^{11} \mathrm{~cm}^{-2}$ are reached, thus in order to qualify as system as the power converter example mentioned above, several systems in parallel need to be tested during several weeks, only possible thanks to the system-level testing design of the facility.

\section{CONCLUSIONS AND OUTLOOK}

We have highlighted the importance of monitoring and simulating the complex radiation field present in the LHC high-energy accelerator and its future upgrade, the HL-LHC. The radiation response of the large quantity of critical COTSbased systems distributed along the accelerator can seriously compromise the operation of the machine. Therefore, measurements and simulations need to be carried out in order to determine the target system SEE cross section and TID and DD lifetime value to ensure an acceptable operation. For future machines this is a complex analysis that requires both the in-depth understanding of the scaling of the radiation source terms, as well as a detailed representation of the accelerator geometry and beam optics. Therefore, tools such as FLUKA provide an essential instrument in the estimation of the energy deposition and radiation levels for future machines such as the HL-LHC.

In the HL-LHC context, depending on the locations near IP1 and IP5, the radiation levels are not only expected to scale proportionally to the number of proton-proton interactions (i.e. integrated luminosity) but will have a further increase owing to e.g. tighter collimator setting or larger leakage of collision debris. This in combination with the large number of COTS-based systems to be installed in the tunnel and shielded alcoves; as well as the demanding limits in terms of number of tolerable radiation failures and TID levels in the DS area, naturally leads to the need of carefully evaluating the RHA of the respective system, and considering their tolerance to radiation from a very early stage of the design. To this regard, the experience and know-how within the R2E project and the availability of a dedicated high-energy mixed-field facility such as CHARM are essential elements to guarantee the success of the system qualification. 


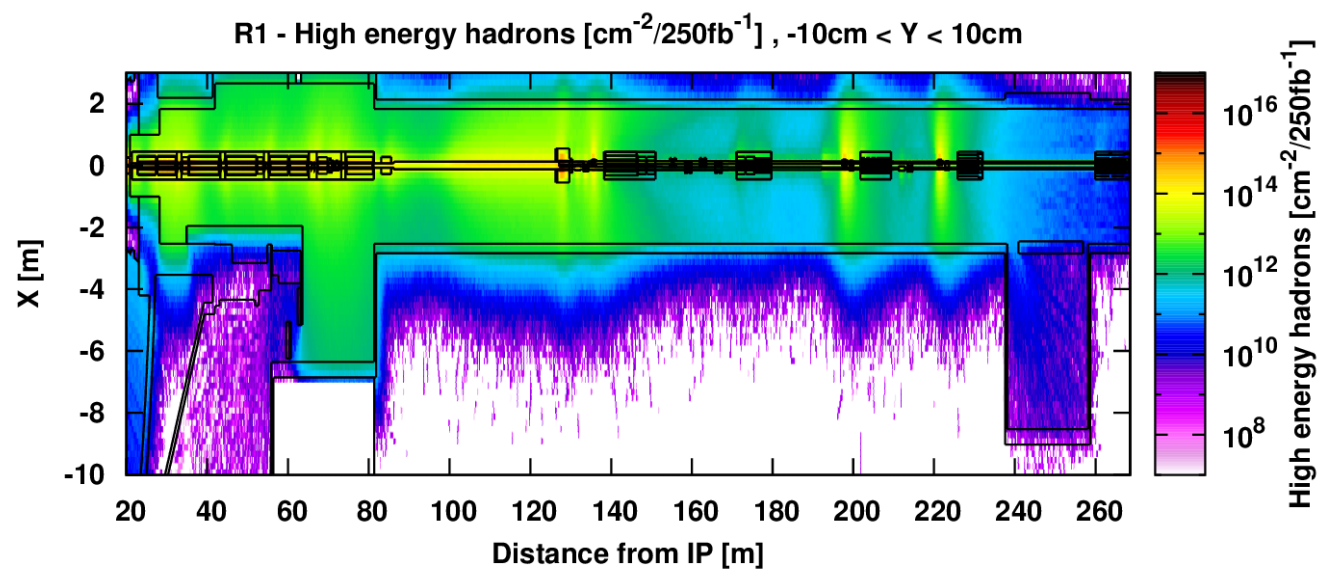

Fig. 14. Simulated annual HL-LHC $\mathrm{HEH}_{\mathrm{eq}}$ fluence right of IP1.

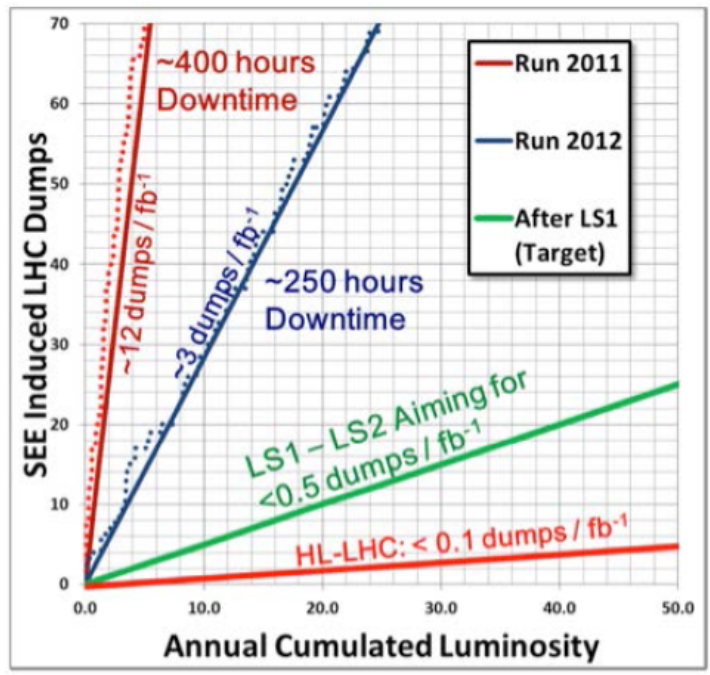

Fig. 15. R2E failures leading to beam dump per unit luminosity. Measured values for 2011-12. For 2015-16, values were slightly better than the target for the LS1-LS2 period.

\section{REFERENCES}

[1] A. Apollonio, M. Brugger, L. Rossi, R. Schmidt, B. Todd, D. Wollmann, and M. Zerlauth, "Roadmap towards High Accelerator Availability for the CERN HL-LHC Era," in Proceedings, 6th International Particle Accelerator Conference (IPAC 2015): Richmond, Virginia, USA, May 3-8, 2015, p. TUPTY053. [Online]. Available: http://accelconf.web.cern.ch/AccelConf/IPAC2015/papers/tupty053.pdf

[2] A. Apollonio, O. R. Orozko, R. Schmidt, M. Valette, D. Wollmann, and M. Zerlauth, "Lessons Learnt from the 2016 LHC Run and Prospects for HL-LHC Availability," in Proc. of International Particle Accelerator Conference (IPAC'17), Copenhagen, Denmark, 14-19 May, 2017, ser. International Particle Accelerator Conference, no. 8. Geneva, Switzerland: JACoW, May 2017, paper TUPVA006, pp. 20392042, https://doi.org/10.18429/JACoW-IPAC2017-TUPVA006. [Online]. Available: http://jacow.org/ipac2017/papers/tupva006.pdf

[3] R. G. Alia, M. Brugger, S. Danzeca, F. Cerutti, J. P. Saraiva, R. Denz, A. Ferrari, L. L. Foro, P. Peronnard, K. Red, R. Secondo, J. Steckert, Y Thurel, I. Toccafondo, and S. Uznanski, "Single event effects in highenergy accelerators," Semiconductor Science and Technology, 2017. [Online]. Available: http://iopscience.iop.org/10.1088/1361-6641/aa5695

[4] L. Evans and P. Bryant, "LHC Machine," Journal of Instrumentation, vol. 3, no. 08, p. S08001, 2008. [Online]. Available: http://stacks.iop.org/1748-0221/3/i=08/a=S08001
[5] W. Herr and B. Muratori, "Concept of luminosity," 2006. [Online]. Available: https://cds.cern.ch/record/941318

[6] G. Apollinari, I. Bejar Alonso, O. Bruening, P. Fessia, M. Lamont, L. Rossi, and L. Tavian, High-Luminosity Large Hadron Collider (HL-LHC): Technical Design Report V. 0.1, ser. CERN Yellow Reports: Monographs. Geneva: CERN, 2017. [Online]. Available: https://cds.cern.ch/record/2284929

[7] A. Lechner et al., "Power Deposition in LHC Magnets With and Without Dispersion Suppressor Collimators Downstream of the Betatron Cleaning Insertion," in Proceedings, 5th International Particle Accelerator Conference (IPAC 2014): Dresden, Germany, June 15-20, 2014. [Online]. Available: http://jacow.org/IPAC2014/papers/mopro021.pdf

[8] B. Dehning, E. Effinger, J. Emery, G. Ferioli, and C. Zamantzas, "An FPGA Based Implementation for Real-Time Processing of the LHC Beam Loss Monitoring System's Data," no. CERN-AB-2007-010, p. 5 p, Nov 2006. [Online]. Available: https://cds.cern.ch/record/1020107

[9] B. Dehning, E. Effinger, J. Emery, G. Ferioli, G. Guaglio, E. B. Holzer, D. Kramer, L. Ponce, V. Prieto, M. Stockner, and C. Zamantzas, "The LHC Beam Loss Measurement System," no. LHC-PROJECT-Report1025., 2007. [Online]. Available: https://cds.cern.ch/record/1057235

[10] C. Martinella et al., "Radiation Levels at the LHC: 2012, 2015 and 2016 Proton Physics Operations in View of HLLHC requirements," in Proc. of International Particle Accelerator Conference (IPAC'17), Copenhagen, Denmark, 14-19 May, 2017, ser. International Particle Accelerator Conference, no. 8. Geneva, Switzerland: JACoW, May 2017, paper TUPVA015, pp. 20752077, https://doi.org/10.18429/JACoW-IPAC2017-TUPVA015. [Online]. Available: http://jacow.org/ipac2017/papers/tupva015.pdf

[11] D. Kramer, M. Brugger, V. Klupak, C. Pignard, K. Roeed, G. Spiezia, L. Viererbl, and T. Wijnands, "LHC RadMon SRAM detectors used at different voltages to determine the thermal neutron to high energy hadron fluence ratio," IEEE Trans. Nucl. Sci., vol. 58, no. 3, pp. 1117 -1122 , june 2011.

[12] G. Spiezia, P. Peronnard, A. Masi, M. Brugger, M. Brucoli, S. Danzeca, R. G. Alia, R. Losito, J. Mekki, P. Oser, R. Gaillard, and L. Dusseau, "A new RadMon version for the LHC and its injection lines," IEEE Trans. Nucl. Sci., vol. 61, no. 6, pp. 3424-3431, Dec 2014.

[13] S. Danzeca, G. Spiezia, M. Brugger, L. Dusseau, G. Foucard, R. G. Alia, P. Mala, A. Masi, P. Peronnard, J. Soltes, A. Thornton, and L. Viererbl, "Qualification and characterization of sram memories used as radiation sensors in the lhc," IEEE Trans. Nucl. Sci., vol. 61, no. 6, pp. 3458-3465, Dec 2014.

[14] K. Roed, M. Brugger, D. Kramer, P. Peronnard, C. Pignard, G. Spiezia, and A. Thornton, "Method for measuring mixed field radiation levels relevant for SEEs at the LHC," IEEE Trans. Nucl. Sci., vol. 59, no. 4 pp. $1040-1047$, Aug. 2012.

[15] O. Stein et al., "Identification and Analysis of Prompt Dose Maxima in the Insertion Regions IR1 and IR5 of the Large Hadron Collider," in Proc. of International Particle Accelerator Conference (IPAC'17), Copenhagen, Denmark, 14-19 May, 2017, ser. International Particle Accelerator Conference, no. 8. Geneva, Switzerland: JACoW, May, 
pp. 2078-2080, https://doi.org/10.18429/JACoW-IPAC2017-TUPVA016. [Online]. Available: http://jacow.org/ipac2017/papers/tupva016.pdf

[16] T. Böhlen, F. Cerutti, M. Chin, A. Fassò, A. Ferrari, P. Ortega, A. Mairani, P. Sala, G. Smirnov, and V. Vlachoudis, "The FLUKA code: developments and challenges for high energy and medical applications," Nuclear Data Sheets, vol. 120, pp. 211-214, 2014.

[17] G. Battistoni, T. Boehlen, F. Cerutti, P. W. Chin, L. S. Esposito, A. Fass, A. Ferrari, A. Lechner, A. Empl, A. Mairani, A. Mereghetti, P. G. Ortega, J. Ranft, S. Roesler, P. R. Sala, V. Vlachoudis, and G. Smirnov, "Overview of the FLUKA code," Annals of Nuclear Energy, vol. 82, pp. $10-18,2015$.

[18] K. Roed, V. Boccone, M. Brugger, A. Ferrari, D. Kramer, E. Lebbos, R. Losito, A. Mereghetti, G. Spiezia, and R. Versaci, "FLUKA simulations for SEE studies of critical LHC underground areas," IEEE Trans. Nucl. Sci., vol. 58, no. 3, pp. 932 -938, June 2011.

[19] G. Battistoni et al., "FLUKA Capabilities and CERN Applications for the Study of Radiation Damage to Electronics at High-Energy Hadron Accelerators," Prog. Nucl. Sci. Tech., vol. 2, pp. 948-954, 2011.

[20] R. Denz, A. Gharib, and D. Hagedorn, "Radiation Resistance and Life Time Estimates at Cryogenic Temperatures of Series produced By-pass Diodes for the LHC Magnet Protection," AIP Conf. Proc., vol. 711, no. LHC-Project-Report-688. CERN-LHCProject-Report-688, pp. 763-770. 9 p, Jan 2003. [Online]. Available: https://cds.cern.ch/record/709124

[21] D. M. Fleetwood and H. A. Eisen, "Total-dose radiation hardness assurance," IEEE Transactions on Nuclear Science, vol. 50, no. 3, pp. 552-564, June 2003.

[22] S. Uznanski, B. Todd, A. Dinius, Q. King, and M. Brugger, "Radiation hardness assurance methodology of radiation tolerant power converter controls for Large Hadron Collider," IEEE Trans. Nucl. Sci., vol. 61, no. 6, pp. 3694-3700, Dec 2014.

[23] J. Mekki, M. Brugger, R. G. Alia, A. Thornton, N. Mota, and S. Danzeca, "CHARM: A mixed field facility at CERN for radiation tests in ground, atmospheric, space and accelerator representative environments," IEEE Transactions on Nuclear Science, vol. 63, no. 4, pp. 2106-2114, Aug 2016.

[24] R. G. Alia, M. Brugger, S. Danzeca, V. Ferlet-Cavrois, C. Frost, R. Gaillard, J. Mekki, F. Saigne, A. Thornton, S. Uznanski, and F. Wrobel, "SEL hardness assurance in a mixed radiation field," IEEE Trans. Nucl. Sci., vol. 62, no. 6, pp. 2555-2562, Dec 2015. 